\title{
SURVEY OF FINE-SCALE STRUCTURE IN THE FAR-INFRARED MILKY WAY
}

\author{
W.H. WALLER ${ }^{1,2,3}$, F. VAROSI ${ }^{1,2,3}$, \\ F. BOULANGER ${ }^{4}$ AND S.W. DIGEL ${ }^{1,3}$ \\ ${ }^{1}$ Hughes STX \\ ${ }^{2}$ StarStuff Inc. \\ ${ }^{3}$ NASA/GSFC \\ ${ }^{4}$ Institut d'Astrophysique Spatiale, Université Paris XI
}

\section{Mapping the Galactic ISM}

What is the general morphology of the diffuse interstellar medium? Is it mostly uniform or clumpy? Are the clumps mostly in the form of spheroidal clouds, sinuous filaments, extended sheets, or discrete shells? And do the clumps or the voids better define the overall structure? By addressing these morphological questions, one can better constrain the dynamical processes that are most responsible for shaping and energizing the ISM.

By mapping the FIR emission from dust that has been warmed by the interstellar radiation field (ISRF), one can trace both the cool and warm phases of the diffuse ISM. These two phases represent most of the mass in the diffuse ISM. Recent data products produced by IPAC from the IRAS mission database provide the best resolved and most complete mapping of the Galactic FIR emission. And through spatial filtering, the strong gradient in brightness towards the Galactic midplane can be eliminated, thereby revealing the fine-scale FIR structure throughout the Galaxy.

\section{Revealing Structure in the FIR Milky Way}

We have produced a $360^{\circ} \times 60^{\circ}$ mural of $100 \mu \mathrm{m}$ emission in the Milky Way from $60^{\circ} \times 60^{\circ}$ mosaics. These mosaics were made from the IRAS Infrared Sky Atlas "plates" using the SkyView Virtual Observatory (found at http://skview.gsfc.nasa.gov/skyview.html). By applying a median normalizing spatial filter, we were able to eliminate the strong gradient in 


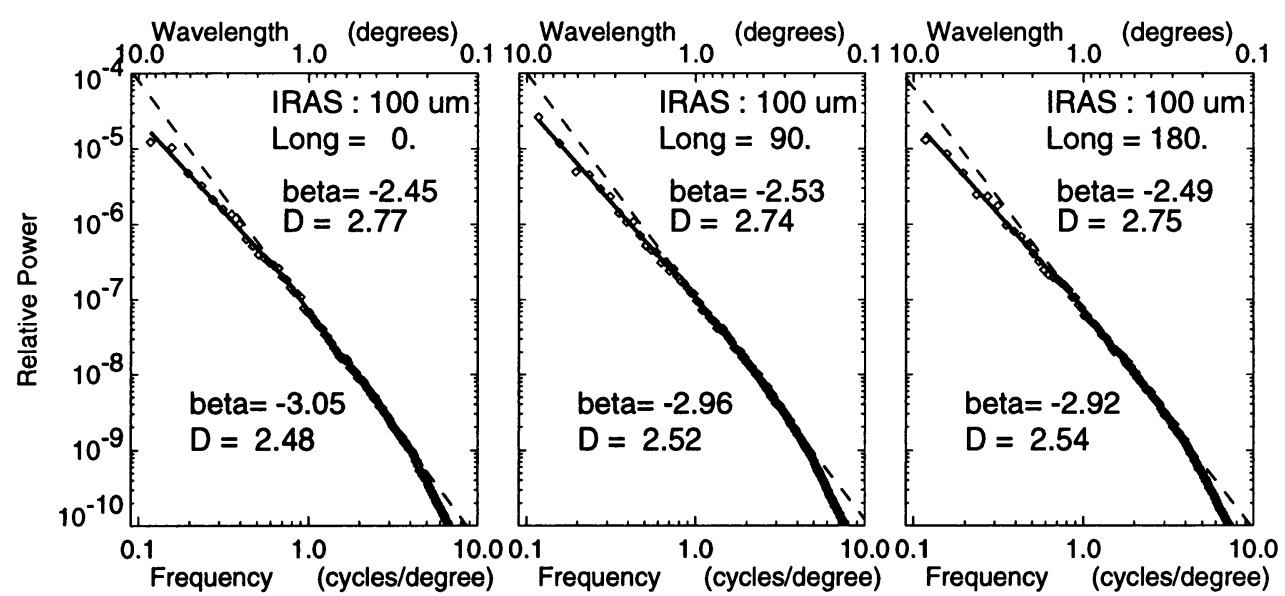

Figure 1. Spatial power spectra of the FIR "froth."

brightness towards the Galactic midplane. The resulting images reveal a "froth" of superposed filaments, voids, and shells (see Figure 2).

This fine-scale structure extends all the way down to the Galactic midplane. Moreover, it scales in intensity with the smoothly varying background, independent of latitude, thus suggesting that the fine-scale residual emission is co-extensive with the smooth background. We conclude that the fine-scale structure is not merely of local origin, but consists of both nearby and more distant features in the disk.

Although we had expected to find morphological evidence for supernovadriven "worms" or "chimneys" rooted in the Galactic plane, our processing shows the FIR fine-scale structure to be more complex (e.g., less coherent and less rooted) as viewed in projection. The observed FIR "froth" is just beginning to be identified with other tracers of the interstellar medium (e.g., $\mathrm{CO}, \mathrm{HI}$, and radio-continuum-cf. Wall and Waller, this volume, p. 191).

Analysis of the spatial statistics shows that the FIR fine-scale structure is self-similar with a angular power-law exponent of $\beta \approx-3$ and and a fractal dimension of $\mathrm{D} \approx 2.5$ - similar to that found in isolated cirrus and molecular clouds. On scales larger than $1.5^{\circ}$, the power-law exponent flattens to $\beta \approx-2.5$, perhaps indicating a change in the characteristic structure (see Figure 1). This could be due to different dynamical inputs organizing the small and large-scale structures (e.g., turbulence and diffusion on small scales vs. macroscopic winds and shock fronts on larger scales).

\section{Acknowledgements}

This research was supported in part by a NASA Astrophysics Data Program contract (\#NAS5-32591) to StarStuff Incorporated. 


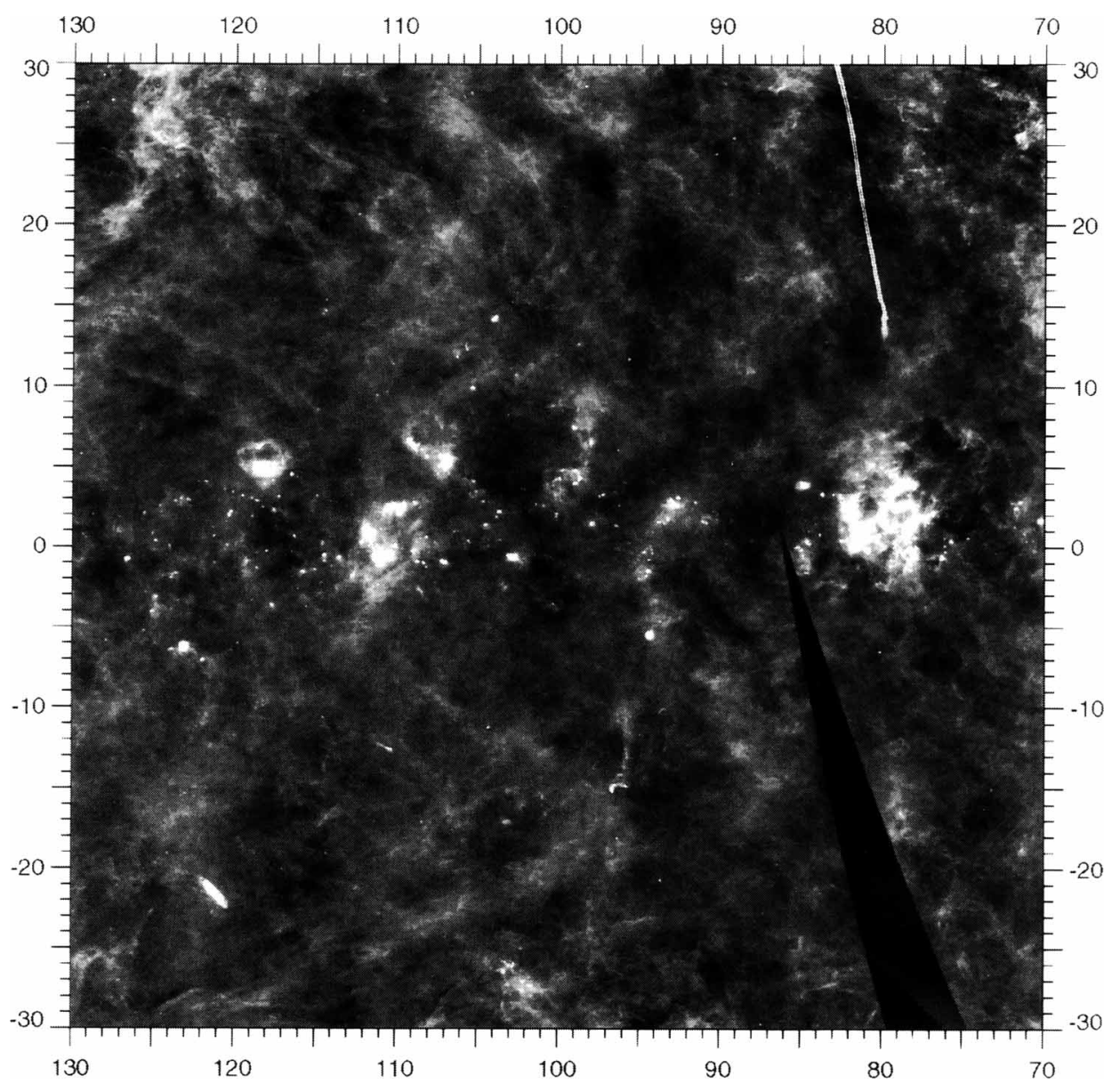

Figure 2. Fine-scale structure in the $100 \mu \mathrm{m}$ emission for the $60^{\circ} \times 60^{\circ}$ field centered at $(\ell, b)=\left(100^{\circ}, 0^{\circ}\right)$. Prominent emitting structures include the Cygnus star-forming region at $(G 78+2)$, IC 1396 at $(G 99+4)$ which seems to form part of a giant shell, NGC 7822 at $(G 118+6)$ which also shows a shell-like morphology, and the galaxy M31 at $(G 121-21)$. The remaining fine-scale features probably represent a superposed mix of nearby and more distant filaments, voids, and shells (see also Wall \& Waller, this volume, p. 191). 